\title{
Oxidized macrophage migration inhibitory factor is a potential new tissue marker and drug target in cancer
}

\author{
Alexander Schinagl ${ }^{1}$, Michael Thiele ${ }^{1}$, Patrice Douillard ${ }^{1}$, Dirk Völkel ${ }^{1}$, Lukas \\ Kenner ${ }^{2,3,4}$, Zahra Kazemi ${ }^{5}$, Michael Freissmuth ${ }^{5}$, Friedrich Scheiflinger ${ }^{1}$, Randolf \\ J. Kerschbaumer ${ }^{1}$ \\ ${ }^{1}$ Baxalta Innovations GmbH, Orth/Donau, Austria \\ ${ }^{2}$ Department of Pathology, Medical University Vienna, Vienna, Austria \\ ${ }^{3}$ Ludwig Boltzmann Institute for Cancer Research, Vienna, Austria \\ ${ }^{4}$ Institute of Laboratory Animal Pathology, University of Veterinary Medicine Vienna, Vienna, Austria \\ ${ }^{5}$ Institute of Pharmacology, Centre of Physiology and Pharmacology, Medical University, Vienna, Austria \\ Correspondence to: Randolf J. Kerschbaumer, email: randolf.kerschbaumer@baxalta.com \\ Keywords: MIF, cancer, inflammation, antibodies, drug target \\ Received: March 18, $2016 \quad$ Accepted: September 02, 2016 \\ Published: September 12, 2016
}

\section{ABSTRACT}

Macrophage migration inhibitory factor (MIF) is a pleiotropic cytokine, which was shown to be upregulated in cancers and to exhibit tumor promoting properties. Unlike other cytokines, MIF is ubiquitously present in the circulation and tissue of healthy subjects. We recently described a previously unrecognized, disease-related isoform of MIF, designated oxMIF, which is present in the circulation of patients with different inflammatory diseases. In this article, we report that oxMIF is also linked to different solid tumors as it is specifically expressed in tumor tissue from patients with colorectal, pancreatic, ovarian and lung cancer. Furthermore, oxMIF can be specifically targeted by a subset of phage display-derived fully human, monoclonal anti-MIF antibodies (mAbs) that were shown to neutralize pro-tumorigenic activities of MIF in vivo. We further demonstrate that anti-oxMIF mAbs sensitize human cancer cell lines (LNCaP, PC3, A2780 and A2780ADR) to the action of cytotoxic drugs (mitoxantrone, cisplatin and doxorubicin) in vitro and in an A2780 xenograft mouse model of ovarian cancer. We conclude that oxMIF is the disease related isoform of MIF in solid tumors and a potential new diagnostic marker and drug target in cancer.

\section{INTRODUCTION}

Macrophage migration inhibitory factor (MIF) is a pro-inflammatory cytokine that promotes tumor growth and metastasis in vivo by multiple modes of action [1-11]. MIF was shown to trigger cell proliferation by activation of the central kinases Akt and ERK, thereby promoting sustained activation and survival of immune cells and cancer cell proliferation [12-14]. Genetic loss of MIF has been described to cause p53-dependent growth alterations, increased p53 transcriptional activity, altered RHOdependent cyclin D1 expression, and resistance to RASmediated oncogenic transformation [15-17]. MIF also plays a key role in angiogenesis and neovascularization: it is associated with hypoxic adaptation and stabilization of hypoxia-inducible factor 1-alpha (HIF-1 $\alpha)$ [6]. In this context, MIF was shown to contribute to the up- regulation of vascular endothelial growth factor (VEGF), IL-8 and matrix metalloproteinases (MMPs) [7, 18, 19]. Furthermore, MIF promotes a pro-inflammatory tumor microenvironment (TME) by induction of cytokines and other mediators of inflammation, such as TNF- $\alpha$ [20], nitric oxide [21] and prostaglandin E2 [12]. Tumor associated macrophages (TAMs) and myeloid-derived suppressor cells (MDSCs) from MIF-deficient mice exhibit reduced immunosuppressive activities resulting in improved immune responses against melanoma [22]. Chemokine functions of MIF are expected to play an important role in altering the TME as they contribute to the infiltration of leukocytes into tumors, thereby promoting cancer related inflammation $[20,23]$.

In vivo, genetic knock-out of MIF was shown to blunt tumor outgrowth in animal models of breast cancer [24], skin cancer [25], gastric cancer [26], bladder cancer 
[27], lung cancer [28] and fibrosarcoma [15]. Blocking of MIF activity either by antibodies or stable RNA interference reduced tumor growth in animal models of colorectal cancer [29], prostate cancer [30], ovarian cancer [20], neuroblastoma [31], pancreatic cancer [10], breast cancer [9], melanoma [22, 32] and lung cancer [28]. The involvement of MIF in human tumor development has been substantiated by reports that describe higher MIF levels in the circulation of cancer patients. Increased circulating MIF levels are correlated with high tumor burden and metastasis in e.g. prostate cancer, lung cancer, colon cancer and ovarian cancer [7, 33-35]. MIF was further shown to be upregulated in the tissue of different tumor types, i.e. pancreatic, breast, prostate, colon, brain, skin, and lung tumors [1, 3, 4, 7, 36-38]. However, MIF cannot be considered a tumor specific marker as it is constitutively expressed and secreted by numerous cell types and significant levels of MIF can be found in the tissue and circulation of healthy subjects [39]. At the first glance, this fact makes MIF a challenging target for specific therapeutic intervention.

We recently reported that MIF occurs in two immunologically distinct redox-dependent isoforms, termed oxidized MIF (oxMIF) and reduced MIF (redMIF) [40]. RedMIF was found to be the abundantly expressed isoform of MIF that can be detected even in healthy subjects. In contrast, oxMIF represents the diseaserelated isoform which was detected predominantly in the circulation and on the surface of cells isolated from patients with inflammatory diseases. The fully human monoclonal anti-oxMIF antibodies BaxB01, BaxG03 and BaxM159 were shown to strictly differentiate between redMIF and oxMIF and to exert in vivo protective effects in animal models of inflammation [40,41]. We therefore sought to investigate the expression of oxMIF in the circulation and in cancer tissue of patients with different types of solid tumors, and to elucidate anti-proliferative effects of oxMIF specific antibodies in combination with cytotoxic drugs.

\section{RESULTS}

\section{OxMIF can be detected in plasma of patients with solid tumors}

Previous studies described the elevation of MIF in the circulation of cancer patients [7, 33-35]. However, these studies did not discriminate between redMIF and oxMIF. We utilized two previously established ELISA methods [40] to quantify oxMIF and total MIF, which reflects the sum of oxMIF and redMIF, in plasma samples of cancer patients and healthy controls. In the control donor group we detected small amounts of oxMIF (up to $10.7 \mathrm{ng} / \mathrm{ml})$ in 20 out of 91 plasma samples $(22 \%$ oxMIF positive; median: $0.0 \mathrm{ng} / \mathrm{ml}$ ) (Figure 1A). OxMIF levels were significantly elevated in plasma samples from patients with ovarian cancer $(23 / 42,55 \%$ oxMIF positive; median: $3.5 \mathrm{ng} / \mathrm{ml}$ ) compared to plasma samples from healthy controls. In plasma samples obtained from patients with prostate cancer $(8 / 14,57 \%$ oxMIF positive; median: $2.4 \mathrm{ng} / \mathrm{ml})$, breast cancer $(8 / 15,53 \%$ oxMIF positive; median: $0.6 \mathrm{ng} / \mathrm{ml})$, head and neck cancer $(27 / 102,26 \%$ oxMIF positive; median: $0.0 \mathrm{ng} / \mathrm{ml}$ ), renal cell carcinoma (13/66, 20\% oxMIF positive; median: $0.0 \mathrm{ng} / \mathrm{ml})$, lung cancer (7/26, 27\% oxMIF positive; median: $0.0 \mathrm{ng} / \mathrm{ml}$ ), colorectal carcinoma $(18 / 140,13 \%$ oxMIF positive; median: $0.0 \mathrm{ng} / \mathrm{ml})$ and pancreatic cancer $(7 / 40,18 \%$ oxMIF positive; median: $0.0 \mathrm{ng} / \mathrm{ml}$ ), levels of oxMIF were not significantly different from the control donor group (Figure 1A). Nevertheless, it is noteworthy that oxMIF levels showed a considerable patient to patient variation, and in each cohort, oxMIF levels $>15 \mathrm{ng} / \mathrm{ml}$ could be found in some individual plasma samples.

As expected, a baseline level of total MIF was detected in the same plasma samples from control donors (median $3.8 \mathrm{ng} / \mathrm{ml}$ ), which is consistent with the MIF levels published in the literature [43, 44]. Significantly increased levels of MIF were detected in plasma from patients with ovarian cancer (median $9.6 \mathrm{ng} / \mathrm{ml}$ ), prostate cancer (median $8.9 \mathrm{ng} / \mathrm{ml}$ ), breast cancer (median $8.4 \mathrm{ng} /$ $\mathrm{ml}$ ), head and neck cancer (median $8.3 \mathrm{ng} / \mathrm{ml}$ ) and renal cell carcinoma (median $10.1 \mathrm{ng} / \mathrm{ml}$ ) (Figure 1B). Total MIF levels of patients with lung cancer, colorectal carcinoma and pancreatic cancer did not differ significantly from those of healthy controls. Correlation plots demonstrated that plasma levels of oxMIF and total MIF correlated in oxMIF positive patients, whereas no correlation was seen in healthy controls (Figure 1C). When oxMIF was detectable, this isoform comprised approximately 55-95\% of MIF in the circulation of patients, which is comparable to the ratio found in patients with inflammatory diseases [40].

Taken together, our data confirm that (total) MIF levels were significantly upregulated in the plasma of patients with solid tumors, such as ovarian cancer [35] or prostate cancer [33]. Circulating oxMIF was significantly elevated in ovarian cancer patients, but not in the other cancer indications tested. Most of the plasma samples from patients with solid tumors did not show detectable amounts of oxMIF. However, oxMIF positive patient subpopulations were identified in all solid tumor indications analyzed, with levels up to $80 \mathrm{ng} / \mathrm{ml}$.

\section{OxMIF occurs specifically in malignant tissue and can be detected in primary tumors and in metastases of different cancers}

MIF has been described to be upregulated in tumor tissue $[1,3,4,7,31,34,36-38]$. Again, these studies did not differentiate between oxMIF and redMIF. Hence, we analyzed tumor tissue from pancreatic, colorectal, ovarian and lung cancer patients for the presence of oxMIF. However, denaturation of MIF - i.e. by fixation 
- leads to irreversible changes in the MIF structure which results in binding of oxMIF specific antibodies and does not allow a differentiation between redMIF and oxMIF [40]. Therefore, conventional immunohistochemistry (IHC) techniques including tissue fixation steps cannot be applied to detect oxMIF in tissue. To avoid this problem we developed an IHC method, which allows for the specific detection of oxMIF, by using fresh frozen tissue sections and avoiding any fixative prior to incubation with anti-oxMIF antibodies. By using this IHC technique, we analyzed tissue derived from patients with pancreatic ductal adenocarcinoma (PDAC). We observed moderate to strong oxMIF immunostaining in pancreatic intraepithelial neoplasias (PanINs) even at an early tumor stage, i.e. stage I-II. Staining was more pronounced in later stage tumors, i.e. stage III, with a prominent staining of the invasion front (Figure 2A, middle and right upper images). Adjacent normal pancreatic tissue did not show immunoreactivity for oxMIF (Figure 2A, upper left image). Unlike oxMIF, total MIF was widely expressed in both, PDAC and adjacent normal pancreas tissue (Figure 2A, lower panel), as expected from the literature [38].
At higher magnification it is obvious that oxMIF was predominantly located at the membrane and the cytoplasm of tumor cells, but was also detected in some nuclei (Figure 2B). Only weak oxMIF staining was detected in the tumor stroma and some infiltrating immune cells like macrophages (Figure 2B).

We next assessed oxMIF expression in tumor tissue from colorectal cancer (CRC) patients, including primary tumors and liver metastases. We observed moderate cytoplasmic and membranous staining for oxMIF in tumor cells and stroma including some nuclei (Figure 2C, middle and right upper images). A pronounced oxMIF staining was also detected in vessel like structures. We therefore probed consecutive slides of CRC liver metastases for cytokeratin 20 (marker for colon epithelial cells), CD31 (endothelial cell marker) and oxMIF. Colocalization of cytokeratin 20 and oxMIF was detected by immunofluorescence microscopy indicating that these oxMIF positive vessel like structures originated from colorectal cancer cells (Figure 2D, lower panel) and were not part of the tumor stroma originating from adjacent liver tissue (Figure 2D, upper panel). Adjacent normal colon
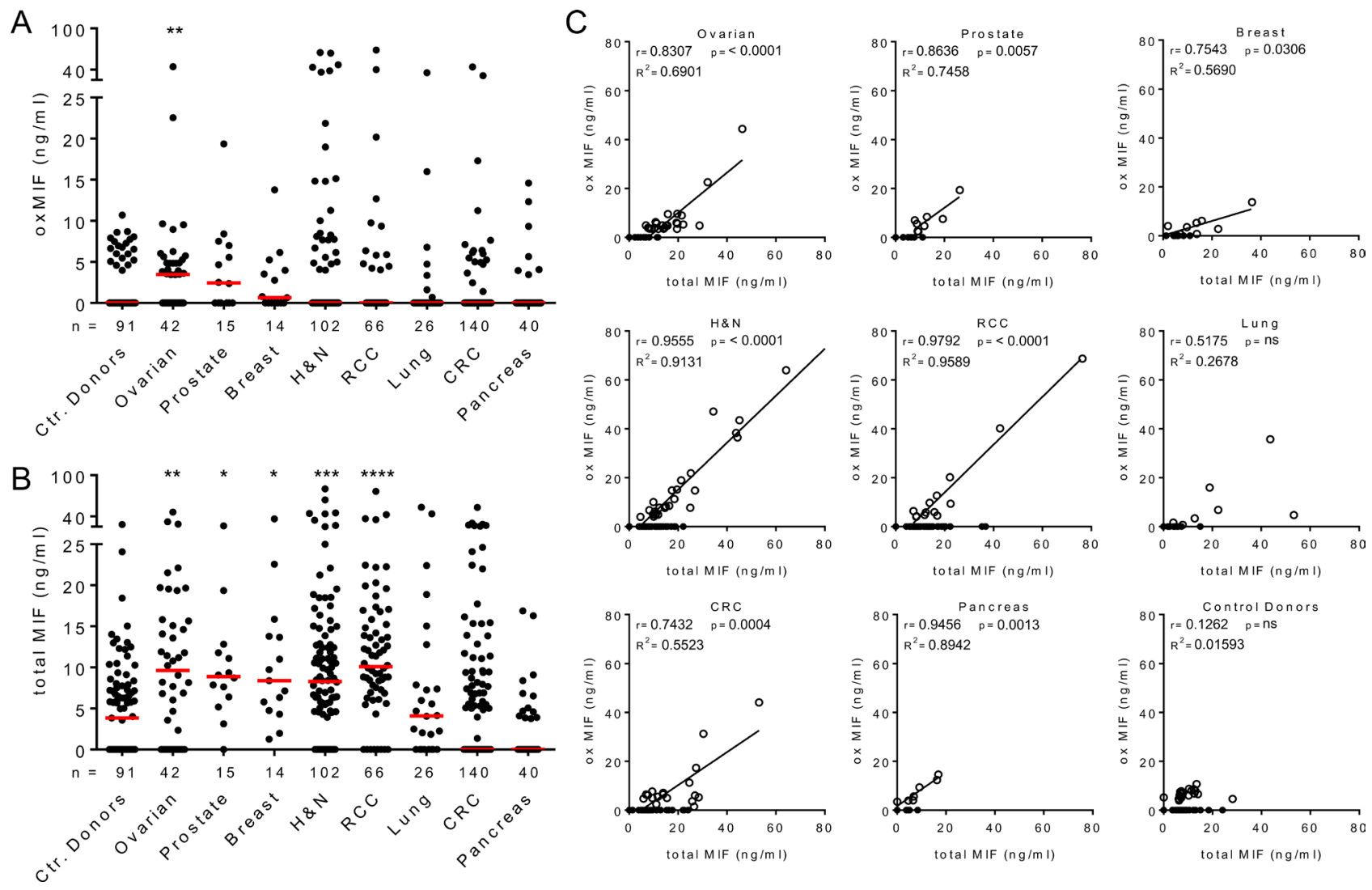

Figure 1: Presence of oxMIF in the circulation of cancer patients and healthy controls. A. Plasma levels of oxMIF in samples from control donors and patients with solid tumors. B. Plasma levels of total MIF in the same samples. Individual values and medians (red lines) are shown. We used the Kruskal-Wallis test followed by Dunn's multiple comparison test for statistical analyses. ${ }^{*} \mathrm{p}<0.05$; $* * \mathrm{p}<0.01 * * * ; \mathrm{p}<0.001 ; * * * * \mathrm{p}<0.0001$. C. OxMIF levels plotted against total MIF levels for each individual plasma sample. OxMIF positive plasma samples (open circles) and oxMIF negative samples (closed circles) are depicted. We used Pearson correlation analysis and linear regression to correlate MIF and oxMIF levels in each cancer type, excluding oxMIF negative samples (oxMIF = $0 \mathrm{ng} / \mathrm{ml})$. 
A

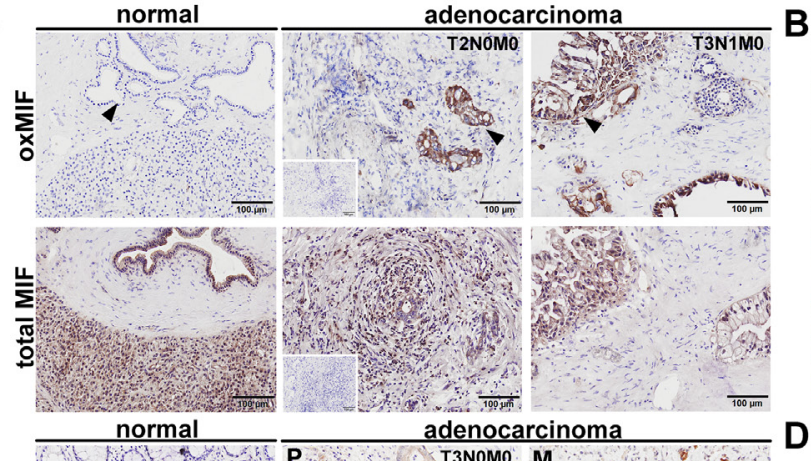

C
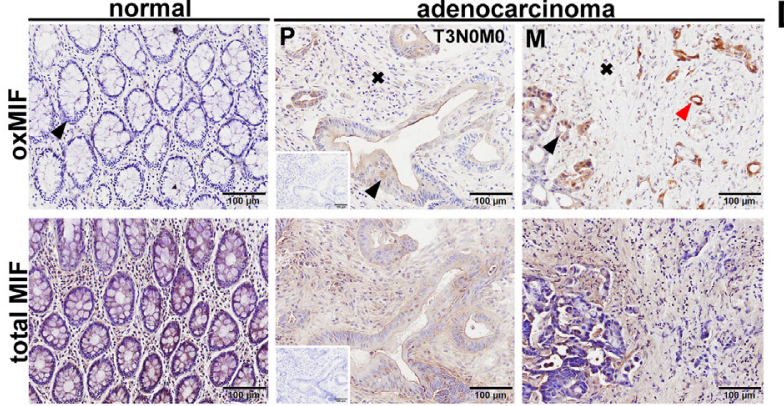

E

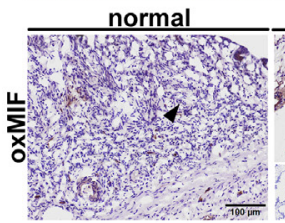

papillary cystadenocarcinoma
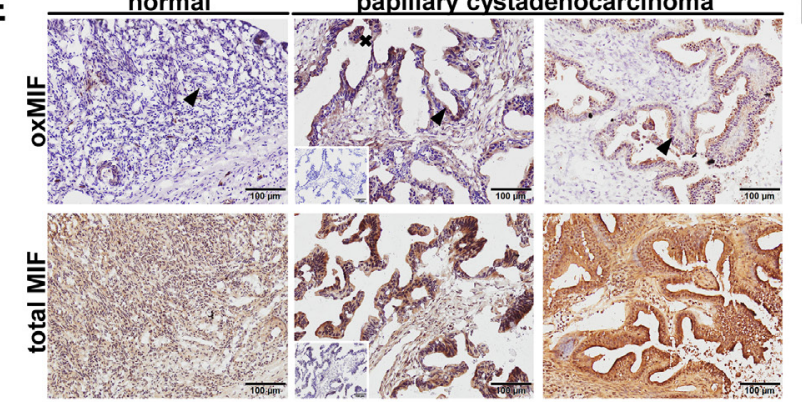

G

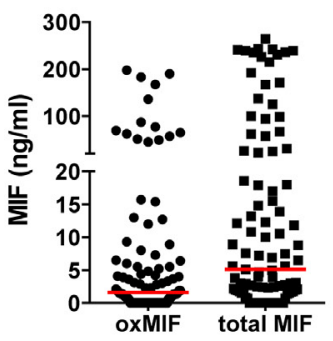

B

H

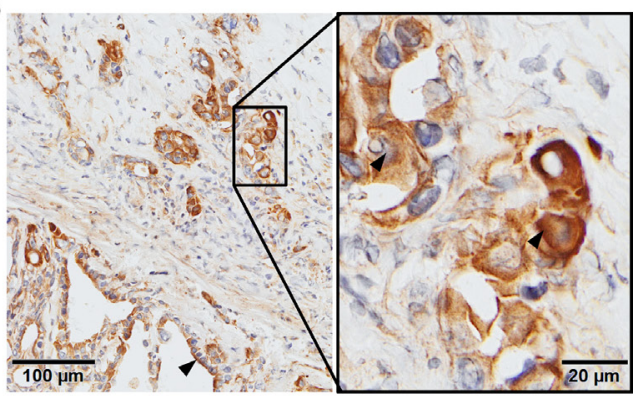

D

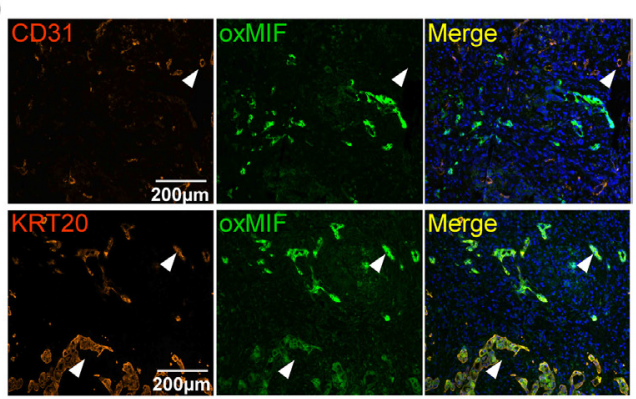

$\mathbf{F}$
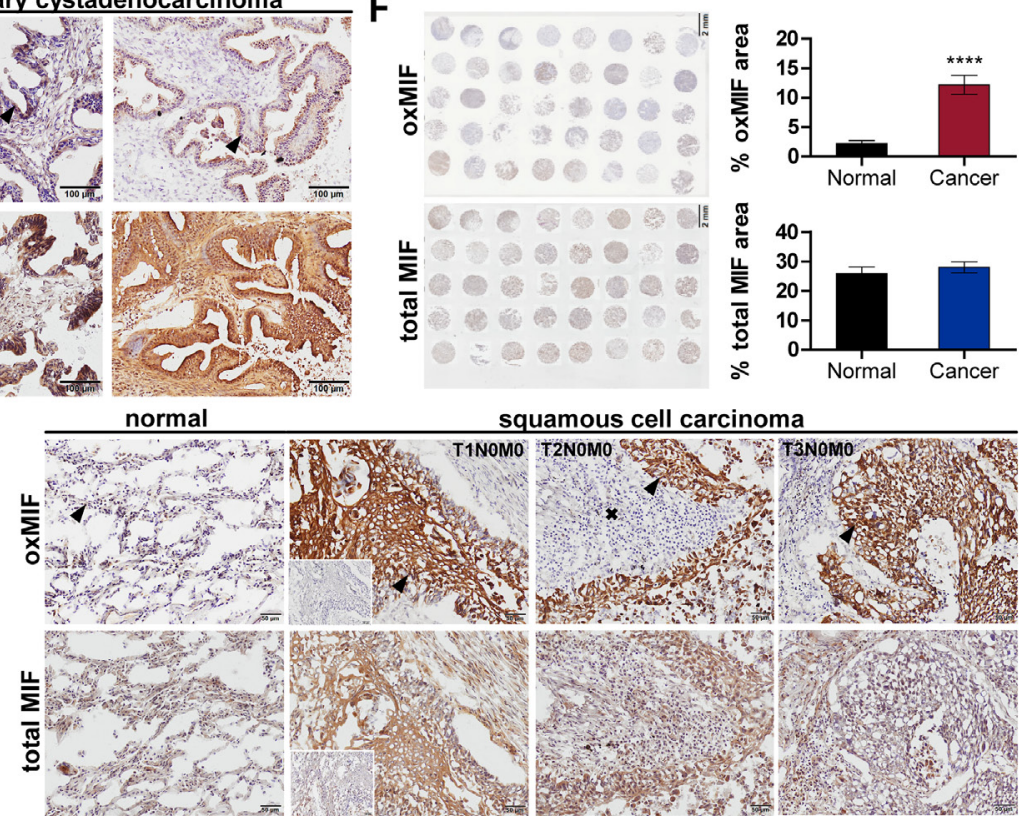

Figure 2: OxMIF occurs specifically in malignant tissue and can be detected in primary tumors and in metastases of different solid tumors. A. OxMIF and total MIF staining of pancreas tissue with normal morphology and pancreatic adenocarcinoma tissue (tumors were staged according to the tumor node metastasis (TNM) system). B. Ten and 40 fold (inset) original magnification of pancreatic adenocarcinoma stained for oxMIF, arrows indicate location of staining (invasion front, membrane, cytoplasm and nucleus, respectively). C. OxMIF and total MIF staining of colon tissue with normal morphology and colorectal adenocarcinoma tissue, red arrows indicate vessel like structures described in D. D. Top panels show immunofluorescence staining of the blood vessel marker CD31 (red) and oxMIF (green). The overlay reveals no co-localization (white arrow) of these markers in CRC metastasis obtained from the liver. Bottom panels show immunofluorescence staining of the colon epithelial cell marker cytokeratin 20 (KRT20, red) and oxMIF (green). The merge with DAPI nuclear counterstain reveals co-localization (white arrow) of these markers. Scale bar $200 \mu \mathrm{m}$. E. OxMIF and total MIF staining of ovarian tissue with normal morphology and ovarian papillary cystadenocarcinoma tissue. F. The left panels show a low magnification image of a tissue micro array consisting of 37 sections of ovarian cancer tissue and 3 adjacent normal tissues, stained for oxMIF and total MIF. Scale bar $2 \mathrm{~mm}$. This microarray has been analyzed by digital images analysis using Definiens Tissue Studio ${ }^{\circledR}$, and the mean stained tissue area \pm SEM are depicted on the right panel. $* * * * p<0.0001$, unpaired two-tailed student's t-test. G. Levels of oxMIF and total MIF in ascitic fluid from patients with ovarian cancer. Data are presented as dot-plot of individual samples with median (red lines). H. OxMIF and total MIF staining of lung tissue with normal morphology and lung cancer tissue as indicated. DAB staining and hematoxylin counterstaining. Scale bars $100 \mu \mathrm{m}$ (if not otherwise indicated). Black arrows and black crosses indicate epithelial cells (in normal tissue) or tumor epithelial cells and tumor stroma respectively, small insets show control staining with matched non-immune isotype IgG. 
mucosa and liver tissue did not show immunoreactivity for oxMIF (Figure 2C, upper left image). In contrast, total MIF was widely expressed in both, CRC (primary and metastatic) derived tissue and adjacent colon mucosa (Figure 2C, lower panel).

We further investigated oxMIF expression in ovarian cancer tissue: weak to strong cytoplasmic and membranous oxMIF staining, depending on the tumor type was observed in apical papillary tumor cells and in cells within the papillary projections as well as in the tumor stroma (Figure 2E, upper panel). The strongest staining was evident in adenocarcinoma, serous adenocarcinoma and mucinous cystadenocarcinoma (Figure 2E and data not shown). OxMIF was not detected in normal ovarian tissue (Figure 2E, upper left image). In addition, we assessed the expression of oxMIF in a customized ovarian cancer tissue micro array (TMA) including 37 ovarian cancer cores and 3 normal ovarian tissue cores. By applying digital image analysis (DIA) single tumor cores were analyzed and the stained tissue area was calculated after accounting for background staining. The result of this DIA demonstrated that oxMIF was significantly overexpressed in ovarian cancer compared to normal ovarian tissue (Figure 2F, upper panel; Supplementary Figure S1). Total MIF showed a moderate to strong uniform staining in each of the tumor cores (tumor cells and stroma) as well as in normal ovarian tissue (Figure 2E, lower panel). DIA revealed no difference in total MIF expression between normal and cancerous tissue (Figure 2F, lower panel; Supplementary Figure S1). We furthermore analyzed ascites fluid from ovarian cancer patients by ELISA to determine the amount of oxMIF and total MIF. OxMIF levels varied from $0 \mathrm{ng} / \mathrm{ml}$ to amounts as high as $200 \mathrm{ng} /$ $\mathrm{ml}(67 / 99,68 \%$ oxMIF positive; median: $1.6 \mathrm{ng} / \mathrm{ml})$. Total MIF levels were slightly higher and ranged from $0 \mathrm{ng} / \mathrm{ml}$ to $260 \mathrm{ng} / \mathrm{ml}$ (median: $5.1 \mathrm{ng} / \mathrm{ml}$ ) (Figure 2G).

Finally, we examined several sections of fresh frozen tumor blocks from non-small cell lung cancer (NSCLC) patients and adjacent non-neoplastic lung epithelium for the presence of oxMIF. We detected oxMIF (weak to strong cytoplasmic staining) in most of the lung cancer samples. Staining intensity varied between the different types of NSCLC, with most prominent staining in adenocarcinomas and squamous cell carcinomas (Figure $2 \mathrm{H}$, upper panel). In some samples, patches of cytoplasmic oxMIF staining were visible in apical tumor cells and oxMIF immunostaining was also apparent in the stroma (Figure 2H, upper panel). For total MIF, we again detected a moderate to strong uniform staining in tumor cells and tumor stroma of most tumor cores as well as in normal lung tissue (Figure 2H, lower panel). No immunoreactivity was seen in tissue sections that were incubated with human or rabbit matched isotype $\mathrm{IgG}$ control antibodies (Figure 2A, 2C, 2E, 2H - insets in middle/second panels).

The IHC analyses demonstrate that oxMIF can be specifically detected in different cancer tissues, whilst it is not detectable in adjacent non-tumorous tissue. Therefore, this study provides new evidence that oxMIF is a promising tissue marker for diagnostic purposes in solid cancers and can be detected prominently at primary tumor sites and corresponding metastases.

\section{Anti-oxMIF mAbs sensitize cancer cells to the action of cytotoxic drugs in vitro and in vivo}

We have previously described that the fully human antibodies BaxB01, BaxG03 and BaxM159 are specific for oxMIF and do not bind to redMIF [40]. We furthermore described that these three antibodies were able to reduce cell growth and viability of prostate cancer cell lines in vitro and in vivo in a monotherapeutic setting [42], whereas a fully human antibody that does not discriminate between oxMIF and redMIF failed to exert significant beneficial anti-tumor effects in vivo (data not shown). Mechanistically, these anti-oxMIF antibodies inhibited proliferation and survival signaling pathways and reduced the level of active ERK1/2 and active Akt, and led to an accumulation of active caspase 3 [42], which is in line with reports on the effects of neutralizing biologic activity of $\operatorname{MIF}[12,14]$.

Due to narrow therapeutic indices, toxicities and development of tumor resistance to chemotherapeutic drugs, current treatment strategies include combinations of targeted therapy with one or more chemotherapeutic agents. Examples include trastuzumab (anti-HER2/neu) in combination with paclitaxel in breast cancer [45], rituximab (anti-CD20) in combination with cyclophosphamide/ doxorubicin/vincristine/dexamethasone in non-Hodgkin's lymphoma [46] or cetuximab (anti-EGFR) in combination with irinotecan in colon cancer [47]. We assessed whether the combination of anti-oxMIF antibody BaxM159 and chemotherapeutics have synergistic effects on tumor cell growth inhibition compared to single agents in vitro and in vivo. As shown in Figure 3, sensitization of the prostate cancer cell lines LNCaP (androgen-receptor positive) or PC3 (androgen-receptor negative) with BaxM159 significantly reduced the half-maximal effective concentration $\left(\mathrm{EC}_{50}\right)$ of mitoxantrone by $40 \%$ and $30 \%$, respectively (Figure $3 \mathrm{~A}$ and Figure $3 \mathrm{~B}$ ). In addition, we found that BaxM159 also sensitized the ovarian cancer cell line A2780 to cisplatin and doxorubicin (= adriamycin). In vitro, BaxM159 reduced the $\mathrm{EC}_{50}$ of cisplatin and doxorubicin by about $70 \%$ and $30 \%$, respectively (Figure $3 \mathrm{C}$ and Figure 3D). Of note, BaxM159 was also able to significantly sensitize the adriamycin-resistant ovarian cancer cell line A2780ADR to the action of doxorubicin by reducing the $\mathrm{EC}_{50}$ by $25 \%$ (Figure $3 \mathrm{E}$ ). We next sought to translate this in vitro synergistic effect of combinatory treatment into an in vivo setting. Mice bearing A2780 ovarian xenografts were treated with $2.5 \mathrm{mg} / \mathrm{kg}$ cisplatin once a week, either in combination with $15 \mathrm{mg} / \mathrm{kg}$ BaxM159 or with $15 \mathrm{mg} / \mathrm{kg}$ isotype control IgG every 
other day. Combination of cisplatin with BaxM159 resulted in significantly reduced final tumor weights $(\sim 45 \%)$ when compared to cisplatin and irrelevant control antibody treated mice (Figure 3F). Our results demonstrate the capability of anti-oxMIF mAbs to sensitize cancer cells to cytotoxic agents which results in an improved antitumorigenic effect.

\section{DISCUSSION}

We recently described the identification of oxMIF, a disease-related isoform of MIF that is predominantly expressed in patients with inflammatory diseases, whereas redMIF represents the ubiquitous isoform of MIF that is abundantly expressed even in healthy subjects. In inflammatory diseases, oxMIF was detected in the circulation of patients, therefore representing a systemic marker of inflammation [40]. In contrast, in cancer indications plasma levels of oxMIF were significantly elevated in samples from ovarian cancer patients, but not in patients with other solid tumor types investigated.

We furthermore described that oxMIF expression is linked to sites of inflammation [40]. This linkage of oxMIF expression to diseased tissue seems to be particularly pronounced in cancer indications, as oxMIF is specifically expressed in the tissue of all human cancer types investigated. Remarkably, oxMIF was detected in tissue of early stage adenocarcinomas of pancreatic and colon cancer, as well as in early stage lung and ovarian tumors. In many ovarian cancer patients oxMIF furthermore accumulated in abdominal ascites, supporting the assumptions that oxMIF expression is linked to the tumor tissue and that oxMIF is released during disease progression. Immunofluorescence analyses of liver metastases proved that oxMIF expression originated from colon epithelial tumor cells. In view of recent findings where MIF was shown to be a key mediator of PDAC metastasis [10], oxMIF is likely to play a role in liver niche formation and $\mathrm{CRC}$ metastasis.

A targeted MIF-directed therapy seems to be challenging due to high MIF plasma levels and its ubiquitous expression in tissue and cells with normal morphology. The specific expression of oxMIF in cancerous tissue allows for targeted inhibition of diseaserelated functions of MIF in cancer. Anti-oxMIF antibodies BaxB01, BaxG03 and BaxM159 were shown to inhibit growth of prostate cancer cells in vitro and in vivo [42]. Here we demonstrated that anti-oxMIF antibody BaxM159 was capable of sensitizing prostate and ovarian cancer cells to the action of cytotoxic drugs in vitro. Combining
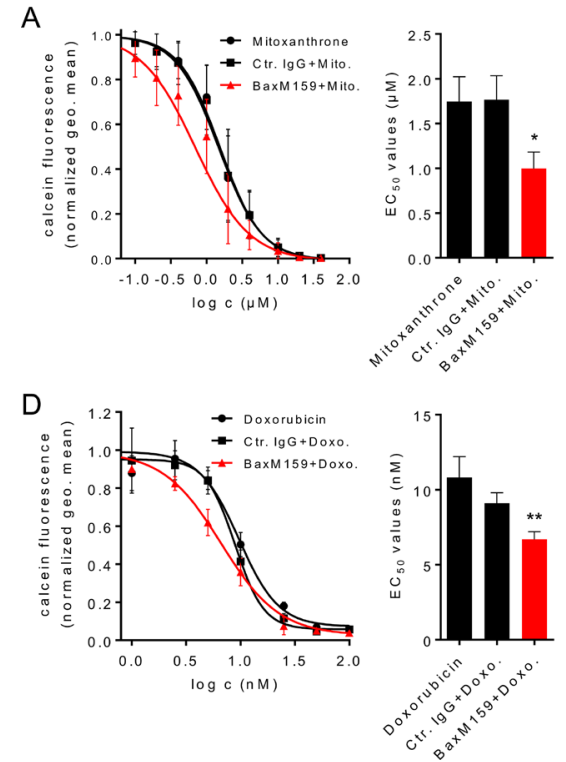

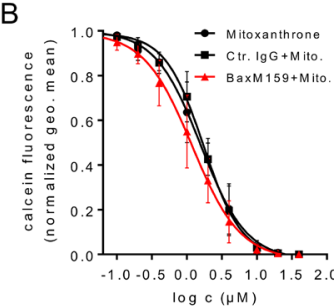

$\log \mathrm{c}(\mu \mathrm{M})$

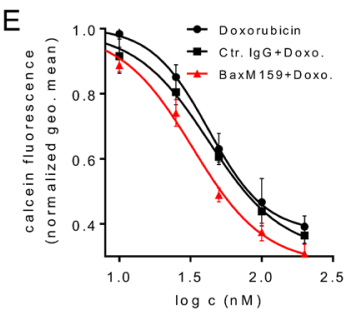

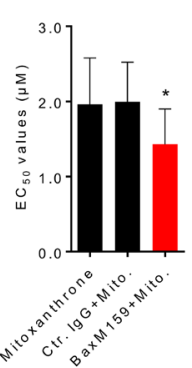

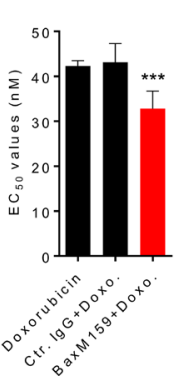

C

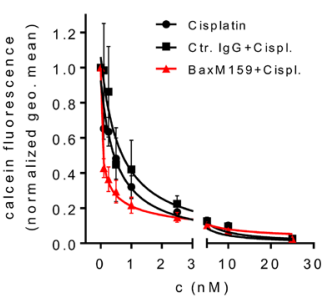

$\mathrm{F}$

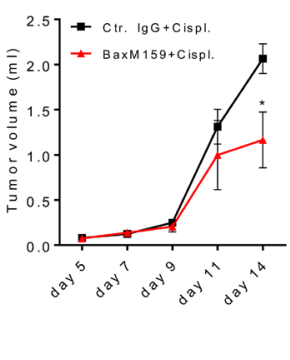

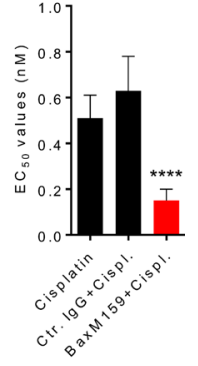

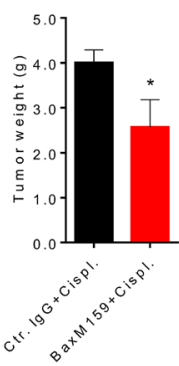

Figure 3: Anti-oxMIF mAbs sensitize cancer cells to cytotoxic drugs in vitro and in vivo. Prostate cancer cell lines $\mathrm{LNCaP}$ A. or PC3 B. were incubated with various concentrations of mitoxantrone $(0.01-40 \mu \mathrm{M})$ either in the presence of $100 \mathrm{nM}$ BaxM159, or matched human isotype control antibody (Ctr. IgG) or without antibody. The ovarian cancer cell line A2780 was incubated with various concentrations of cisplatin (0.1-25 nM) C. or doxorubicin (3.13-200 nM) D. either in the presence of $50 \mathrm{nM}$ BaxM159, or matched human isotype control antibody ( $\mathrm{Ctr}$. IgG) or without antibody. The adriamycin-resistant ovarian cancer cell line A2780ADR E. was incubated with various concentrations of doxorubicin (3.13-200 nM) either in the presence of $50 \mathrm{nM}$ BaxM159, or matched human isotype control antibody (Ctr. IgG) or without antibody. After $48 \mathrm{~h}$ cells were labeled with calcein-AM and live cells were counted by flow cytometry. EC50 values for cytotoxic drugs were calculated by fitting the data points to a four-parameter variable slope equation (Hill-equation). Curve fits (left panels) and EC50 values (right panels) are represented as means \pm SEM from at least 4 independent experiments. ${ }^{*} \mathrm{p}<0.05 ;{ }^{*} \mathrm{p}<0.01$; $* * * \mathrm{p}<0.001 ; * * * * \mathrm{p}<0.0001$. We used one-way ANOVA followed by Dunnett's multiple comparison test. F. MF-1 nude mice ( $\mathrm{n}=10$ /group) were inoculated with $1 \times 10^{6}$ A2780 cells suspended in matrigel. Mice were treated with cisplatin $(2.5 \mathrm{mg} / \mathrm{kg}) \mathrm{and} \mathrm{BaxM} 159(15 \mathrm{mg} / \mathrm{kg}) \mathrm{or}$ human $\mathrm{Ctr}$. $\operatorname{IgG}(15 \mathrm{mg} / \mathrm{kg})$. Tumor volumes were measured at indicated time points. On day 14, mice were sacrificed, the tumors excised and weighed. Data are shown as means \pm SEM. ${ }^{*} \mathrm{p}<0.05$. We used student's unpaired t-test. 
cisplatin with BaxM159 also increased the cytotoxicity of cisplatin in vivo, which resulted in reduced A2780 tumor growth rates in mice. A fully human anti-MIF antibody which does not distinguish between redMIF and oxMIF failed to exert a significant beneficial effect in a mouse xenograft prostate cancer model (results not shown). This is in line with the previously reported observation that recombinant human antibodies that do not discriminate between the two isoforms failed to show beneficial therapeutic effects in animal models of inflammation [40].

Reduction of the level of activated kinases Akt and ERK1/2 and activation of caspases were previously described as underlying mechanisms for the anti-tumor effects of anti-oxMIF antibodies [42]. As oxMIF could be detected on the cellular surface and in the cytoplasm of tumor cells, it will be of interest to discover whether anti-oxMIF antibodies interfere with extracellular and/ or intracellular functions of MIF which convert into an inhibition of Akt and ERK1/2 signaling and in an activation of caspases. An interference of anti-oxMIF antibodies with intrinsic MIF activities is conceivable as an increased caspase activity could be mediated not only via Akt but also via the MIF-p53 axis [15]. Anti-oxMIF antibodies induce neither complement-dependent cell lysis nor antibody-dependent cellular cytotoxicity. Therefore, the beneficial effect of down regulation of these kinases might have been enhanced by the direct cytotoxic effects of cisplatin, doxorubicin and mitoxantrone. It will also be important to identify immunomodulatory activities of anti-oxMIF antibodies in the tumor microenvironment as processes of stromal remodeling could be crucial for an increased cytotoxicity of chemotherapeutics when administered in combination with anti-oxMIF antibodies. An interference of anti-oxMIF antibodies with proangiogenic properties of MIF might also be a part of the mechanism of action and should therefore be addressed in future investigations. Furthermore, the exact structural basis for the rearrangement of redMIF to the diseased related isoform oxMIF as well as MIF binding proteins and receptors that are involved in regulating this transition are currently unknown and require further investigations.

Taken together, we demonstrated that oxMIF is a new therapeutic target in solid tumors and that anti-oxMIF antibodies are a new class of potent inhibitors of MIFrelated functions in tumorigenesis with a promising use in combination therapy. We also highlight oxMIF as a compelling new tissue marker which might be important for diagnostic and prognostic purposes, especially in combination with treatment strategies.

\section{MATERIALS AND METHODS}

\section{Reagents}

BaxB01, BaxM159, and an isotype-matched human control antibody were produced as described [41].
Monoclonal rabbit anti-MIF antibodies were generated by immunization of rabbits with recombinant MIF, spleens were resected and B-cells were used for RabMabHybridomatechnology (Epitomics, Abcam). High affinity hybridoma clones were selected and RabMab IgGs were purified from cell culture supernatant over protein A columns. Recombinant MIF was expressed in E. coli and purified as described [41]. Mitoxantrone, cisplatin and doxorubicin were obtained as commercial formulations approved for human use $\left(\right.$ Ebexantron $^{\circledR}$, Platinol $^{\circledR}$, Adriblastin $^{\circledR}$ ).

\section{Human sample collection}

Plasma samples from healthy subjects (control donors) were collected from volunteers in a normal state of health, with no apparent signs of disease. Plasma samples from control donors and cancer patients were purchased either from Tissue Solutions Ltd., Asterand Bioscience Inc., Cureline Inc. or Biochemed Services Inc. Frozen tissue samples and frozen tissue micro arrays were acquired from Asterand Bioscience Inc. and Biochain Inc., respectively. All patients and control donors signed an informed consent for sample collection.

\section{A2780 ovarian cancer xenograft mouse model}

Human ovarian cancer cells A2780 were harvested from exponentially growing cultures and mixed with growth factor-depleted matrigel (BD); $1 \times 10^{6}$ cells in $0.25 \mathrm{ml}$ matrigel were then inoculated subcutaneously into the right flank of female MF1 nude mice. One day after inoculation, treatment ( $\mathrm{n}=10$ in each animal group) was started by i.p. administration of the indicated doses of BaxM159 and control antibody. Repetitive administration of antibodies was done every other day. Cisplatin was administered i.p. once per week at a dose of $2.5 \mathrm{mg} / \mathrm{kg}$. Treatment with cisplatin was started one day after the first antibody administration. The sizes of tumor xenografts were measured when their growth became evident (typically on day 7) and volumes were calculated using the formula $\mathrm{V}=0.5^{*} \mathrm{a} * \mathrm{~b}^{2}$ (where " $\mathrm{a}$ " is the longest diameter and " $b$ " is the shortest diameter). Animal experiments were carried out in accordance with the guidelines set forth by the Medical University of Vienna (MUW) (Good Scientific Practice Manual) and were approved by the Animal Welfare Committee of the Medical University of Vienna (Tierversuchskommission) and the Austrian Ministry of Science and Research.

\section{Cellular assays}

Cultures of human prostate cancer cell lines PC3 and LNCaP as well as of the human ovarian cancer cell lines A2780 and A2780ADR were maintained in RPMI 1640 medium supplemented with both $10 \%$ fetal calf serum and $2 \mathrm{mM}$ glutamine. Cells were grown at $37^{\circ} \mathrm{C}$ 
in a humidified incubator with $5 \% \mathrm{CO}_{2}$. The indicated concentrations of mitoxantrone, cisplatin or doxorubicin and/or antibodies were added and the cells were incubated for $48 \mathrm{~h}$. Thereafter, the cells were detached with Accutase $^{\circledR}$, pelleted by centrifugation and resuspended in ice cold phosphate-buffered saline (PBS) containing 50 nM calcein-AM. Flow cytometry $(10,000$ events recorded per measurement; $n=3$ per determination) was performed on a Becton Dickinson FACScan ${ }^{\circledR}$ with forward scatter, sideward scatter and calcein fluorescence recorded in individual channels. Analysis was done by WinMDI $2.9^{\circledR}$ software. The geometric mean of the calcein fluorescence was extracted and used as the parameter for further statistical analysis.

\section{ELISAs}

Quantitative determination of oxMIF and total MIF was done as described previously [40].

\section{Immunohistochemistry}

To detect oxMIF, fresh frozen tissue slides were air dried, blocked (BB: $20 \%$ goat serum / 2\% BSA / 0.2\% fish gelatin) and incubated with biotinylated primary antibody (biotinylated BaxB01 or biotinylated non-immune human $\mathrm{IgG}$, both $5 \mu \mathrm{g} / \mathrm{ml})$ diluted in TBS containing $2 \%(\mathrm{w} / \mathrm{v})$ BSA and $0.2 \%(\mathrm{w} / \mathrm{v})$ fish gelatin (PADB). The slides were fixed in $10 \%(\mathrm{w} / \mathrm{v})$ PBS buffered formalin solution (VWR) and permeabilized in $0.1 \%$ TritonX-100 in TBS for prior addition of the enzyme block solution (Dual Endogenous Enzyme Block, Dako). Sections were incubated with RTU Vectastain ABC reagent (Vector Laboratories) and developed with ImmPact DAB substrate (Vector Laboratories). Alternatively, non-labeled BaxB01 (1 $\mu \mathrm{g} /$ $\mathrm{ml}$, prior-fixation) and monoclonal rabbit-anti-BaxB01 antibodies $(0.5 \mu \mathrm{g} / \mathrm{ml})$ in combination with goat antirabbit-HRP (Thermo Scientific, 31460, 1:500) were used to detect oxMIF. Following chromogenesis, the sections were washed in tap water, counterstained with hematoxylin (Vector Laboratories), dehydrated in ethanol, cleared in xylene and coverslips were mounted with Vecta ${ }^{\circledR}$ Mount permanent mounting medium (Vector Laboratories).

To detect total MIF, fresh frozen tissue slides were fixed in 10\% PBS buffered formalin (VWR) and permeabilized by incubating the sections with $0.1 \%$ TritonX-100 in TBS. Endogenous peroxidases were blocked by incubating the tissue sections with dual endogenous peroxidase block (Dako). Unspecific binding was blocked with BB. The sections were incubated with monoclonal rabbit anti-MIF antibodies $(1 \mu \mathrm{g} / \mathrm{ml})$ or commercial polyclonal rabbit anti-MIF antibodies (FL115 Santa Cruz; 1:200) and rabbit IgG isotype control diluted in PADB. The staining was done using goat anti-rabbit-HRP (Thermo Scientific) diluted in PADB or ImmPRESS HRP Anti-Rabbit Ig (Vector Laboratories). The Liquid DAB + Substrate Chromogen System (Dako) was used for chromogenic reaction. Counterstaining and mounting was done as described above. Full slide scans were acquired using an Olympus VS120 slide scanning microscope at 20-fold magnification (UPLANSAPO 20x, NA 0.75; PIKE F505/C Camera, Allied Vision Technologies). Pictures are presented at 10 -fold original magnification. Analyses and evaluation and quantification of stainings were performed by a board certified pathologist (L. K.). Digital image analysis (DIA) was performed with Definiens Tissue Studio ${ }^{\circledR}$ v3.6 program.

\section{Immunofluorescence}

Fresh frozen tissue slides were air dried, blocked with BB and incubated with BaxB01 (4 $\mu \mathrm{g} / \mathrm{ml}$, in PDAB) for oxMIF detection. The specimen was fixed in $10 \%$ PBS buffered formalin (VWR). Furthermore, the sections were incubated with monoclonal mouse anti-Cytokeratin 20 (Dako M7019, 1:50) or anti-CD31 antibodies (e-bioscience 13-0319, 1:200), and monoclonal rabbit antiBaxB01 antibodies $(0.5 \mu \mathrm{g} / \mathrm{ml})$ diluted in PADB $+0.25 \%$ TritonX-100. Rabbit and mouse antibodies were detected by Alexa Fluor ${ }^{\circledR}$ conjugated secondary antibodies (Life Technologies A11034 and A21424, 1:2000) diluted in $\mathrm{PADB}+0.25 \%$ TritonX-100. The slides were rinsed in PBS and coverslips were mounted with ProLong Gold Antifade reagent with DAPI (Life Technologies). Pictures were taken at 20-fold magnification (LUCPlanFLN 20x, NA 0.45 ) with an Olympus inverted microscope (VS81) using a mercury lamp and the filters DAPI (U-MWU2), FITC (U-MWIBA3) and TRITC (U-MWIGA3), and a XM10 camera (Olympus).

\section{Statistics}

Distributions were evaluated by KolmogorovSmirnov test. If normal distribution was confirmed, data were evaluated by one-way ANOVA followed by Dunnett's multiple comparison. Otherwise data were evaluated by Kruskal-Wallis test followed by Dunn's multiple comparison. Two groups were compared by unpaired two-tailed student's t-test (normal distribution) or Mann-Whitney-test. Correlation analysis was done using Pearson correlation analysis and linear regression.

\section{CONFLICTS OF INTEREST}

A. S., M. T., P. D., D. V., F. S. and R. J. K. are full time employees of Baxalta. F. S. and R. J. K. have ownership interest (including patents). M.F. received a research grant from Baxalta's predecessor Baxter. M.F. and L.K. are consultants of Baxalta.

\section{REFERENCES}

1. Bando H, Matsumoto G, Bando M, Muta M, Ogawa T, Funata N, Nishihira J, Koike M, Toi M. Expression of 
macrophage migration inhibitory factor in human breast cancer: association with nodal spread. Jpn J Cancer Res. 2002; 93:389-396.

2. Kamimura A, Kamachi M, Nishihira J, Ogura S, Isobe H, Dosaka-Akita H, Ogata A, Shindoh M, Ohbuchi T, Kawakami Y. Intracellular distribution of macrophage migration inhibitory factor predicts the prognosis of patients with adenocarcinoma of the lung. Cancer. 2000; 89:334-341.

3. Markert JM, Fuller CM, Gillespie GY, Bubien JK, McLean LA, Hong RL, Lee K, Gullans SR, Mapstone TB, Benos DJ. Differential gene expression profiling in human brain tumors. Physiol Genomics. 2001; 5:21-33.

4. Meyer-Siegler K, Fattor RA, Hudson PB. Expression of macrophage migration inhibitory factor in the human prostate. Diagn Mol Pathol. 1998; 7:44-50.

5. Takahashi N, Nishihira J, Sato Y, Kondo M, Ogawa H, Ohshima T, Une Y, Todo S. Involvement of macrophage migration inhibitory factor (MIF) in the mechanism of tumor cell growth. Mol Med. 1998; 4:707-714.

6. Winner M, Leng L, Zundel W, Mitchell RA. Macrophage migration inhibitory factor manipulation and evaluation in tumoral hypoxic adaptation. Methods Enzymol. 2007; 435:355-369.

7. He XX, Chen K, Yang J, Li XY, Gan HY, Liu CY, Coleman TR, Al-Abed Y. Macrophage migration inhibitory factor promotes colorectal cancer. Mol Med. 2009; 15:1-10.

8. Fingerle-Rowson G, Kaleswarapu DR, Schlander C, Kabgani N, Brocks T, Reinart N, Busch R, Schutz A, Lue H, Du X, Liu A, Xiong H, Chen Y, Nemajerova A, Hallek $\mathrm{M}$, Bernhagen J, et al. A tautomerase-null macrophage migration-inhibitory factor (MIF) gene knock-in mouse model reveals that protein interactions and not enzymatic activity mediate MIF-dependent growth regulation. Mol Cell Biol. 2009; 29:1922-1932.

9. Simpson KD, Templeton DJ, Cross JV. Macrophage migration inhibitory factor promotes tumor growth and metastasis by inducing myeloid-derived suppressor cells in the tumor microenvironment. J Immunol. 2012; 189:5533-5540.

10. Costa-Silva B, Aiello NM, Ocean AJ, Singh S, Zhang H, Thakur BK, Becker A, Hoshino A, Mark MT, Molina H, Xiang J, Zhang T, Theilen TM, Garcia-Santos G, Williams $\mathrm{C}$, Ararso Y, et al. Pancreatic cancer exosomes initiate premetastatic niche formation in the liver. Nat Cell Biol. 2015; 17:816-826.

11. Hu CT, Guo LL, Feng N, Zhang L, Zhou N, Ma LL, Shen L, Tong GH, Yan QW, Zhu SJ, Bian XW, Lai MD, Deng YJ, Ding YQ. MIF, secreted by human hepatic sinusoidal endothelial cells, promotes chemotaxis and outgrowth of colorectal cancer in liver prometastasis. Oncotarget. 2015; 6:22410-22423. doi:10.18632/oncotarget.4198.

12. Mitchell RA, Metz CN, Peng T, Bucala R. Sustained mitogen-activated protein kinase (MAPK) and cytoplasmic phospholipase A2 activation by macrophage migration inhibitory factor (MIF). Regulatory role in cell proliferation and glucocorticoid action. J Biol Chem. 1999; 274:18100-18106.

13. Lue H, Kapurniotu A, Fingerle-Rowson G, Roger T, Leng L, Thiele M, Calandra T, Bucala R, Bernhagen J. Rapid and transient activation of the ERK MAPK signalling pathway by macrophage migration inhibitory factor (MIF) and dependence on JAB1/CSN5 and Src kinase activity. Cell Signal. 2006; 18:688-703.

14. Lue H, Thiele M, Franz J, Dahl E, Speckgens S, Leng L, Fingerle-Rowson G, Bucala R, Luscher B, Bernhagen J. Macrophage migration inhibitory factor (MIF) promotes cell survival by activation of the Akt pathway and role for CSN5/JAB1 in the control of autocrine MIF activity. Oncogene. 2007; 26:5046-5059.

15. Fingerle-Rowson G, Petrenko O, Metz CN, Forsthuber TG, Mitchell R, Huss R, Moll U, Muller W, Bucala R. The p53dependent effects of macrophage migration inhibitory factor revealed by gene targeting. Proc Natl Acad Sci U S A. 2003; 100:9354-9359.

16. Petrenko O, Fingerle-Rowson G, Peng T, Mitchell RA, Metz CN. Macrophage migration inhibitory factor deficiency is associated with altered cell growth and reduced susceptibility to Ras-mediated transformation. J Biol Chem. 2003; 278:11078-11085.

17. Swant JD, Rendon BE, Symons M, Mitchell RA. Rho GTPase-dependent signaling is required for macrophage migration inhibitory factor-mediated expression of cyclin D1. J Biol Chem. 2005; 280:23066-23072.

18. Ren Y, Chan HM, Li Z, Lin C, Nicholls J, Chen CF, Lee PY, Lui V, Bacher M, Tam PK. Upregulation of macrophage migration inhibitory factor contributes to induced N-Myc expression by the activation of ERK signaling pathway and increased expression of interleukin-8 and VEGF in neuroblastoma. Oncogene. 2004; 23:4146-4154.

19. Li Z, Ren Y, Wu QC, Lin SX, Liang YJ, Liang HZ. Macrophage migration inhibitory factor enhances neoplastic cell invasion by inducing the expression of matrix metalloproteinase 9 and interleukin- 8 in nasopharyngeal carcinoma cell lines. Chin Med J (Engl). 2004; 117:107-114.

20. Hagemann T, Robinson SC, Thompson RG, Charles K, Kulbe H, Balkwill FR. Ovarian cancer cell-derived migration inhibitory factor enhances tumor growth, progression, and angiogenesis. Mol Cancer Ther. 2007; 6:1993-2002.

21. Bernhagen J, Mitchell RA, Calandra T, Voelter W, Cerami A, Bucala R. Purification, bioactivity, and secondary structure analysis of mouse and human macrophage migration inhibitory factor (MIF). Biochemistry. 1994; 33:14144-14155.

22. Yaddanapudi K, Putty K, Rendon BE, Lamont GJ, Faughn JD, Satoskar A, Lasnik A, Eaton JW, Mitchell RA. Control of tumor-associated macrophage alternative activation by 
macrophage migration inhibitory factor. J Immunol. 2013; 190:2984-2993

23. Li J, Mo HY, Xiong G, Zhang L, He J, Huang ZF, Liu ZW, Chen QY, Du ZM, Zheng LM, Qian CN, Zeng YX. Tumor microenvironment macrophage inhibitory factor directs the accumulation of interleukin-17-producing tumorinfiltrating lymphocytes and predicts favorable survival in nasopharyngeal carcinoma patients. J Biol Chem. 2012; 287:35484-35495.

24. Schulz R, Marchenko ND, Holembowski L, FingerleRowson G, Pesic M, Zender L, Dobbelstein M, Moll UM. Inhibiting the HSP90 chaperone destabilizes macrophage migration inhibitory factor and thereby inhibits breast tumor progression. J Exp Med. 2012; 209:275-89.

25. Martin J, Duncan FJ, Keiser T, Shin S, Kusewitt DF, Oberyszyn T, Satoskar AR, VanBuskirk AM. Macrophage migration inhibitory factor (MIF) plays a critical role in pathogenesis of ultraviolet-B (UVB) -induced nonmelanoma skin cancer (NMSC). FASEB J. 2009; 23:720-730.

26. Wilson JM, Coletta PL, Cuthbert RJ, Scott N, MacLennan K, Hawcroft G, Leng L, Lubetsky JB, Jin KK, Lolis E, Medina F, Brieva JA, Poulsom R, Markham AF, Bucala R, Hull MA. Macrophage migration inhibitory factor promotes intestinal tumorigenesis. Gastroenterology 2005; 129:1485-503.

27. Taylor JA, 3rd, Kuchel GA, Hegde P, Voznesensky OS, Claffey K, Tsimikas J, Leng L, Bucala R, Pilbeam C. Null mutation for macrophage migration inhibitory factor (MIF) is associated with less aggressive bladder cancer in mice. BMC Cancer. 2007; 7:135.

28. Mawhinney L, Armstrong ME, C OR, Bucala R, Leng L, Fingerle-Rowson G, Fayne D, Keane MP, Tynan A, Maher L, Cooke G, Lloyd D, Conroy H, Donnelly SC. Macrophage migration inhibitory factor (MIF) enzymatic activity and lung cancer. Mol Med. 2014; 20:729-735.

29. Ogawa H, Nishihira J, Sato Y, Kondo M, Takahashi $\mathrm{N}$, Oshima T, Todo S. An antibody for macrophage migration inhibitory factor suppresses tumour growth and inhibits tumour-associated angiogenesis. Cytokine. 2000; 12:309-314.

30. Meyer-Siegler KL, Iczkowski KA, Leng L, Bucala R, Vera PL. Inhibition of macrophage migration inhibitory factor or its receptor (CD74) attenuates growth and invasion of DU-145 prostate cancer cells. J Immunol. 2006; 177:8730-8739.

31. Ren Y, Chan HM, Fan J, Xie Y, Chen YX, Li W, Jiang GP, Liu Q, Meinhardt A, Tam PK. Inhibition of tumor growth and metastasis in vitro and in vivo by targeting macrophage migration inhibitory factor in human neuroblastoma. Oncogene. 2006; 25:3501-3508.

32. Ioannou K, Cheng KF, Crichlow GV, Birmpilis AI, Lolis EJ, Tsitsilonis OE, Al-Abed Y. ISO-66, a novel inhibitor of macrophage migration, shows efficacy in melanoma and colon cancer models. Int J Oncol. 2014; 45:1457-1468.
33. Meyer-Siegler KL, Iczkowski KA, Vera PL. Further evidence for increased macrophage migration inhibitory factor expression in prostate cancer. BMC Cancer. 2005; 5:73.

34. Tomiyasu M, Yoshino I, Suemitsu R, Okamoto T, Sugimachi K. Quantification of macrophage migration inhibitory factor mRNA expression in non-small cell lung cancer tissues and its clinical significance. Clin Cancer Res. 2002; 8:3755-3760.

35. Krockenberger M, Kranke P, Hausler S, Engel JB, Horn E, Nurnberger K, Wischhusen J, Dietl J, Honig A. Macrophage migration-inhibitory factor levels in serum of patients with ovarian cancer correlates with poor prognosis. Anticancer Res. 2012; 32:5233-5238.

36. Shimizu T, Abe R, Nakamura H, Ohkawara A, Suzuki M, Nishihira J. High expression of macrophage migration inhibitory factor in human melanoma cells and its role in tumor cell growth and angiogenesis. Biochem Biophys Res Commun. 1999; 264:751-758.

37. Howard BA, Zheng Z, Campa MJ, Wang MZ, Sharma A, Haura E, Herndon JE, 2nd, Fitzgerald MC, Bepler G, Patz EF, Jr. Translating biomarkers into clinical practice: prognostic implications of cyclophilin A and macrophage migratory inhibitory factor identified from protein expression profiles in non-small cell lung cancer. Lung Cancer. 2004; 46:313-323.

38. Tan L, Ye X, Zhou Y, Yu M, Fu Z, Chen R, Zhuang B, Zeng B, Ye H, Gao W, Lin Q, Li Z, Zhou Q. Macrophage migration inhibitory factor is overexpressed in pancreatic cancer tissues and impairs insulin secretion function of beta-cell. J Transl Med. 2014; 12:92.

39. Calandra T, Roger T. Macrophage migration inhibitory factor: a regulator of innate immunity. Nat Rev Immunol. 2003; 3:791-800.

40. Thiele M, Kerschbaumer RJ, Tam FW, Volkel D, Douillard P, Schinagl A, Kuhnel H, Smith J, McDaid JP, Bhangal G, Yu MC, Pusey CD, Cook HT, Kovarik J, Magelky E, Bhan A, et al. Selective Targeting of a Disease-Related Conformational Isoform of Macrophage Migration Inhibitory Factor Ameliorates Inflammatory Conditions. J Immunol. 2015; 195:2343-2352.

41. Kerschbaumer RJ, Rieger M, Volkel D, Le Roy D, Roger T, Garbaraviciene J, Boehncke WH, Mullberg J, Hoet RM, Wood CR, Antoine G, Thiele M, Savidis-Dacho H, Dockal M, Ehrlich H, Calandra T, et al. Neutralization of macrophage migration inhibitory factor (MIF) by fully human antibodies correlates with their specificity for the beta-sheet structure of MIF. J Biol Chem. 2012; 287:7446-7455.

42. Hussain F, Freissmuth M, Volkel D, Thiele M, Douillard P, Antoine G, Thurner P, Ehrlich H, Schwarz HP, Scheiflinger F, Kerschbaumer RJ. Human anti-macrophage migration inhibitory factor antibodies inhibit growth of human prostate cancer cells in vitro and in vivo. Mol Cancer Ther. 2013; 12:1223-1234. 
43. Emonts M, Sweep FC, Grebenchtchikov N, Geurts-Moespot A, Knaup M, Chanson AL, Erard V, Renner P, Hermans PW, Hazelzet JA, Calandra T. Association between high levels of blood macrophage migration inhibitory factor, inappropriate adrenal response, and early death in patients with severe sepsis. Clin Infect Dis. 2007; 44:1321-1328.

44. Mizue Y, Nishihira J, Miyazaki T, Fujiwara S, Chida M, Nakamura K, Kikuchi K, Mukai M. Quantitation of macrophage migration inhibitory factor (MIF) using the one-step sandwich enzyme immunosorbent assay: elevated serum MIF concentrations in patients with autoimmune diseases and identification of MIF in erythrocytes. Int J Mol Med. 2000; 5:397-403.

45. Slamon DJ, Leyland-Jones B, Shak S, Fuchs H, Paton V, Bajamonde A, Fleming T, Eiermann W, Wolter J, Pegram M,
Baselga J, Norton L. Use of chemotherapy plus a monoclonal antibody against HER2 for metastatic breast cancer that overexpresses HER2. N Engl J Med. 2001; 344:783-792.

46. Coiffier B. Rituximab in combination with $\mathrm{CHOP}$ improves survival in elderly patients with aggressive non-Hodgkin's lymphoma. Tumori. 2002; 88:S26-28.

47. Sobrero AF, Maurel J, Fehrenbacher L, Scheithauer W, Abubakr YA, Lutz MP, Vega-Villegas ME, Eng C, Steinhauer EU, Prausova J, Lenz HJ, Borg C, Middleton G, Kroning H, Luppi G, Kisker O, et al. EPIC: phase III trial of cetuximab plus irinotecan after fluoropyrimidine and oxaliplatin failure in patients with metastatic colorectal cancer. J Clin Oncol. 2008; 26:2311-2319. 